\section{Contribution of organic acids to lake acidity}

SIR-We would like to reply to the correspondence by Krug', who raised criticisms of a recent paper ${ }^{2}$ of ours. In that paper, we quantified the contributions of organic acids to lakewater acidity, in the simplest case by comparisons of lakes with similar concentrations of base cations and sulphate but differing in total organic carbon (TOC). The contribution of dissociated organic acids is 4-5 $\mu \mathrm{eq} \mathrm{mg}^{-1} \mathrm{TOC} \mathrm{l}^{-1}$, a value shown to be independent of $\mathrm{Ca}^{2+}$, $\mathrm{SO}_{4}^{2-}$ and $p \mathrm{H}$ when $p \mathrm{H} \sim 5-7$; the value decreases to $2.5-3.5 \mu \mathrm{eq} \mathrm{mg}^{-1}$ TOC $^{-1}$ in lakes with $p \mathrm{H}<4.8$ (ref. 3 ).

We measured contributions of organic acids to strong and weak acidity. The average $p \mathrm{~K}$ of organic acids is 3.5-5.0 (ref. 4) and there were approximately equal quantities of strong and weak organic acids. The range of $p \mathrm{Ks}$ indicates that there is no relationship between $p \mathrm{H}$ and $\mu$ eq of charge per mg TOC for $p \mathrm{H}>5$. The organic acids cannot be entirely weak acids as claimed by $\mathrm{Krug}^{1}$, but act as $p \mathrm{H}$ buffers as sulphate concentration increases, particularly if $\mathrm{H}_{2} \mathrm{SO}_{4}$ is added to water that has a $p \mathrm{H}$ near the $p \mathrm{~K}$ of abundant organic acids.

Krug cites palaeolimnological data for Lake Hovvatn in southern Norway to support his hypothesis that strong mineral acid replaces organic acid on an equivalent basis, but he fails to address the magnitude of the total changes in lakewater chemistry. Whether or not pre-acidification TOC was 2-3 times higher than now is unimportant, but the absolute magnitude of changes in organic anion, base cation, $\mathrm{H}^{+}$and aluminium concentrations are significant. A change in $p \mathrm{H}$ from 5.2-5.4 to 4.4 represents a $34-\mu$ eq $\mathrm{I}^{-1}$ increase in $\mathrm{H}^{+}$, and this has been accompanied by an increase in $\mathrm{Al}$ of $\sim 20 \mu \mathrm{eq} \mathrm{l}^{-1}$. Krug has therefore underestimated both the $\mathrm{Al}$ concentration (which influences fish mortality) and the total change in acidity. Studies of diatom reconstructions of lakewater $p \mathrm{H}$ indicate that $p \mathrm{H}$ changes are associated with atmospheric deposition $^{5.6}$.

Krug misquotes the value of the factor $F$, the normalized estimated anthropogenic $\mathrm{SO}_{4}^{2-}$ input (ref. 7). Henriksen ${ }^{7}$ did not propose 0.4 as a regional value, but rather demonstrated that this was the maximum possible value at current acidity levels for concentrations $\mathrm{Ca}^{*}+\mathrm{Mg}^{*}=0$; the value $F=0.2$ was proposed as being realistic, in the absence of further data. Clearly, $F$ is a function of base cation concentration, and must approach zero in the most dilute lakes. Neither the organic buffering hypothesis nor the $F$ factor discount the validity of our regression analysis or those of others ${ }^{\mathrm{x}}$.

The units of conductivity in our paper were $\mathrm{mS} \mathrm{m} \mathrm{m}^{-1}$ rather than $\mu \mathrm{S} \mathrm{cm} \mathrm{cm}^{-1}$. The data are of high quality and the ionic contributions balance.

The empirical data on lakes and streams indicate a correlation between the location of acidic clearwater lakes and the spatial and temporal occurrence of elevated aluminium levels and acid deposition. Krug cannot explain why such lakes are found only in areas of Norway that receive acid rain but not in areas with lower deposition of sulphate. Elevated sulphate levels also lower the $p \mathrm{H}$ of humic lakes in areas receiving acid deposition below that from organic acidity.

Institute for Watershed Studies, David F. BrakKe

Western Washington University, Bellingham, Washington 98225, USA

Arne Henriksen Norwegian Institute for Water Research, PO Box 33, Blindern,

N-0313 Oslo, Norway

Stephen A. Norton

Department of Geological Sciences,

University of Maine,

Orono, Maine 04469, USA

1. Krug, E.C. Nature 334, 571 (1988).

2. Brakke, D.F., Henriksen, A. \& Norton, S.A. Nature 329 432-434 (1987)

3. Henriksen, A., Brakke, D.F. \& Norton, S.A. Envir. Sci Technol. (in the press)

Perdue, E.M., Reuter, J.H. \& Parrish, R.S. Geochim cosmochim. Acta 48, 1257-1263(1984)

5. Davis, R.B. Quat. Sci. Rev. 6, 147-163 (1987)

6. Flower, R.J., Battarbee, R.W. \& Appleby, P.G. J. Ecol 75, 797 -824 (1987)

Henriksen, A. Verh. int. Verein. Limnol. 22, 692-698 (1984); Acid Ruin Res. Rep. no. 1 (NIVA, Oslo, 1982) 8. Gorham, E. et al. Nature 324, 451-453 (1986).

\section{Two chimps for HIV}

Sir-The 'two chimpanzees' approach endorsed in HIV research by Prince et al. (Nature 333, 513; 1988) is challenged by Holland (Nature 334, 478; 1988) on statistical grounds. But it is the only accessible approach, and provided one knows its limitations it is reasonable and indispensable. The numbers calculated by Holland are so high that they would have precluded the development of hepatitis B vaccines. Yet correct inferences were drawn continuously from very small numbers of chimpanzees during the development and production of hepatitis $B$ vaccines.

With HIV, the 'two chimpanzees' approach has shown that several candidate antigens fail to protect against experimental HIV infection, and it has been correctly concluded that one cannot expect significant protection of man against HIV with these formulations. Any others that are developed should be shown to protect at least two chimpanzees before being studied in man. HIV-infected chimpanzees need stricter and lifelong infectious containment than do hepatitisB-infected chimpanzees - an additional reason for their parsimonious use

JAN DESMYTER

Rega Institute for Medical Research,

$B-3000$ Leuven, Belgium
Radiation hazards in space put in perspective

SIR-Now that timetables are under consideration for further visits to the Moon and for an exploratory mission to Mars, the Commentary by Letaw et al. ${ }^{1}$ on the radiation hazards of space travel should be put into perspective.

The radiation environments in space are certainly less benign than on Earth, but Letaw et al. paint an unnecessarily gloomy picture for missions beyond the magnetosphere. They have raised important questions about the shielding and secondary radiations generated by the interaction of the primary particles and the shielding material, as well as the dangers of solar particle events. We are concerned that they may have given the impression that the dangers from radiation, especially from heavy ions and the infrequent very large solar particle events, are not only frighteningly large but known with confidence.

As Letaw et al.' aver, little is known as yet about the effects of heavy ions on humans, so information must be gleaned from experiments with animal models. These authors state that because of the comparative deposition of energy "a rare cosmic-iron nucleus is 13,500 times as dangerous as a cosmic ray proton". Yet, the available assessments of the comparative biological dangers of radiations of different qualities have not been based on fluence but on dose. The relationships between comparative energy deposition and comparative danger are complex. Relative biological effectiveness (RBE), based on dose, for a number of endpoints, such as life shortening, cataract and cancer, is in the range of 1 to about 30 (refs 2-8; E. Blakely, personal communication).

Furthermore, it should be noted that the fluence of iron particles is low. Not more than about one per cent of galactic cosmic rays are heavy ions. In low Earth orbits the contribution of galactic cosmic rays to the total dose equivalent is small. For example, in the orbit proposed for the Space Station the galactic cosmic ray dose equivalent is estimated to be about $7 \mathrm{mSv}$ $(0.7 \mathrm{rem})$ for a ninety-day mission, or a little more than twice the average annual effective dose equivalent from various sources of natural background radiation on Earth'. This is not to imply that the radiation levels are of no concern. They are of concern but they are manageable.

Apart from the effects of galactic cosmic rays and the secondary radiations in missions beyond the magnetosphere, the authors state that "rare solar particle events could deliver a lethal dose of up to 1,000 rem in one day". Such high dose equivalents could be incurred but, to our knowledge, only in the skin. Major contributors to such high surface doses are lowenergy protons that do not penetrate into 